\title{
Composites
}

\section{Confinement-induced polymorphism in acetylsalicylic acid-nanoporous glass composites}

\author{
P. Peksa ${ }^{1,2}$ (D) J. Trzmiel ${ }^{2}$ (D), M. Ptak $^{3}$ (D), M. Kostrzewa ${ }^{1}$ (D), R. Szatanik ${ }^{4}$ (D) A. Barascu $^{5}$ (D), D. Enke ${ }^{5}$ (D), \\ and A. Sieradzki ${ }^{2, *}$ (D) \\ ${ }^{1}$ Faculty of Physics, Opole University of Technology, Ozimska 75, 45-370 Opole, Poland \\ ${ }^{2}$ Faculty of Fundamental Problems of Technology, Wrocław University of Science and Technology, Wybrzeże Wyspiańskiego 27, \\ 50-370 Wrocław, Poland \\ ${ }^{3}$ Institute of Low Temperature and Structure Research, Polish Academy of Sciences, Box 1410, 50-950 Wrocław 2, Poland \\ ${ }^{4}$ Institute of Physics, Opole University, Oleska 48, Opole 45-052, Poland \\ ${ }^{5}$ Institute of Chemical Technology, Universität Leipzig, 04103 Leipzig, Germany
}

Received: 13 June 2018

Accepted: 21 August 2018

Published online:

30 August 2018

(C) The Author(s) 2018

\begin{abstract}
We report on the experimental observation of confinement-induced new phase appearance in acetylsalicylic acid (ASA)-porous glass (PG) composites. In this study, ASA was embedded in PG host matrices of various pore widths (15-200 nm). The Raman spectra and positron annihilation lifetime measurements exhibit the existence of ASA nanocrystals in the PG matrix. The DSC data revealed that the melting temperature $T_{M}$ and excess specific heat decrease with decreasing the size of embedded ASA nanocrystals. The close inspection of the $T_{\mathrm{M}}$ dependence versus diameter of filled pores has shown that the ASA crystallizes in polymorph II in confined matrix. Moreover, it was demonstrated that the ASA spatial confinement results in the appearance of new polymorphic phase in the investigated composites-highly likely form ASA III. Both the changes in ASA melting temperatures due to the volume constraints and the decrease in specific heat may be crucial for the bioavailability of the drug.
\end{abstract}

\section{Introduction}

Aspirin (acetylsalicylic acid) is one of the most popular drugs used in a broad therapeutic range. It belongs to the group of non-steroidal anti-inflammatory drugs. Simple chemical reaction-acetylation, which is an example of non-enzymatic modification of proteins [1], lies at the core of its action within the human body. Aspirin has been known for many years. The analgesic properties of the salicylate-rich willow bark were already appreciated by Hippocrates and medieval herbalists. In 1899, Hoffmann obtained a stable and pure derivative of salicylic acid, i.e., acetylsalicylic acid (ASA) [2]. ASA is obtained by reacting salicylic acid with acetic anhydride in the presence of sulfuric acid or phosphoric acid as a

Address correspondence to E-mail: adam.sieradzki@pwr.edu.pl 
catalyst [3]. Moreover, it is worth mentioning that aspirin is prone to hydrolysis [4].

Polymorphic substances exist in different crystalline structures. They find applications in various fields. Among other things, this group of substances includes pharmaceuticals, minerals and water [5]. Polymorphism of ASA was unclear until 2004, when it was predicted and the structure of its second polymorphic phase was described in the following year $[6,7]$. This finding triggered very intense studies on ASA polymorphism, which is one of its most interesting properties [8].

Currently, the metastable phase of ASA is being intensively studied [9]. Form I ASA is dimethyloxymetric dimeric acid molecules connected by centrosymmetric methyl $\mathrm{C}-\mathrm{H} \cdots \mathrm{O}$ hydrogen bonds. It was recently shown [10] that crystalline ASA forms two distinct types (polymorphs)-form I and form II [10], which differ solely in one elementary unit cell dimension. It was confirmed that the second, multilayer type is metastable and can be converted into its original form through a one-dimensional shear-slip mechanism [10, 11]. It has also been proven that the activation energy barrier increases under pressure [12]. This explains previous experimental observations on ASA II compression, which does not return to ASA I over time [12]. Single phase II ASA crystals can be obtained by crystallization of aspirin in the presence of anhydrous ASA in organic solvents $[11,12]$. DSC measurements revealed the difference in melting phase transition temperature at $135.5^{\circ} \mathrm{C}$ for phase II and at $144.9^{\circ} \mathrm{C}$ for phase I [13].

Besides phases I and II, the existence of phases III and IV has been experimentally proved [14] and the structure of ASA was determined by a combination of X-ray powder diffraction analysis and crystal structure prediction algorithms [15]. High-pressure investigations have revealed that phase III appeared to be the most stable polymorphic form of aspirin. It was shown that above the pressure of $2 \mathrm{GPa}$ phase I ASA is not directly converted into the stable form II, but into a new phase III aspirin [16]. Discovery of the ASA III polymorph was a consequence of observations of the form of crystals from the melt. In the case of the investigated polymorph melt, crystallization was stated to be less common than solution crystallization [17]. It has also been presented that ASA II limits molecules to a higher-energy conformer. Therefore, it may be concluded that under high pressure the ASA II is being transformed into the
ASA I. What is more, at a pressure above $7 \mathrm{GPa}$ structural distortion of these two ASA phases should be expected [14].

In the structure of ASA IV, the plane of the acetyl group is nearly perpendicular to aryl ring plane than forms ASA I and ASA II. However, the orientation of the group $\mathrm{OH}$ of the carboxyl moiety is the far side with respect to the acetyl group, so it is in the gas phase conformation [6]. Moreover, ASA IV characterized by higher potential energy may lead to faster bioavailability [18].

Nanoporous matrices embedded with various crystalline solids have been studied for decades. The behavior of polymorphic materials has usually been studied after placing them in controlled host materials such as nanoporous aluminum or silicon membranes with pore diameters from 1 to $100 \mathrm{~nm}$. Interestingly, during such confinement, common trends can be observed, which are similar for a variety of different matrices and guest molecules [19]. The classical nucleation theory assumes that the phase stability of the porous matrix is determined by the balance between the surface free energy and stabilizing volume free energy. The size constraint is imposed by nano-sized pores during crystallization and large surface area to volume ratios. These constraints are reflected in the resulting crystal properties. The differences may occur, e.g., in melting points or enthalpy. Moreover, confining the material in the nanoporous host system can significantly affect the crystallized phases and the crystalline polymorphism [19].

The objective of the paper was to investigate physical properties of acetylsalicylic acid-porous glass composites (ASA-PG) with different pore sizes using independent experimental techniques. ASA was confined in porous matrices of various pores sizes. (Diameter ranges from 15 to $200 \mathrm{~nm}$.) We have investigated the influence of the ASA nanocrystals diameter confined into porous matrices on its melting temperature. Usually, at ambient conditions solely stable form I ASA is observed. However, we have shown that the spatial confinement of ASA has an impact on its structural stability. Thus, in the presented study not only the presence of form I ASA, but also form II and III ASA was detected. 


\section{Experimental}

\section{Porous glass matrix}

For the preparation of the porous glass (PG) matrices, two various initial glass compositions were used, i.e., $70 \mathrm{SiO}_{2} \cdot 23 \mathrm{~B}_{2} \mathrm{O}_{3} \cdot 7 \mathrm{Na}_{2} \mathrm{O}$ (wt\%) (glass I) and $62.5 \mathrm{SiO}_{2} \cdot 30.5 \mathrm{~B}_{2} \mathrm{O}_{3} \cdot 7 \mathrm{Na}_{2} \mathrm{O}$ (wt\%) (glass II). The glasses were melted from reagent-grade chemicals $\left(\mathrm{SiO}_{2}\right.$, Alfa Aesar 99.5\%, $\mathrm{B}_{2} \mathrm{O}_{3}$, Alfa Aesar $99 \%$ and $\mathrm{Na}_{2} \mathrm{CO}_{3}, \mathrm{BDH}$ Prolabo $100 \%$ Ph. Eur.) at $1500{ }^{\circ} \mathrm{C}$ in an electrical furnace (Nabertherm HT40/17) in a $\mathrm{Pt} 80 / \mathrm{Rh} 20$ crucible for $2 \mathrm{~h}$. Subsequently, the melted glasses were poured in a preheated $\left(480{ }^{\circ} \mathrm{C}\right)$ ashlarformed brass mold with the dimensions of $10 \times 20 \times 100 \mathrm{~mm}$. To avoid stress in the glass, the solidified glass blocks were placed into another furnace at $480{ }^{\circ} \mathrm{C}$ and slowly cooled down to room temperature. For the preparation of the samples with 15, 25, 65 and $90 \mathrm{~nm}$ median pore width, glass I was thermally treated for $24 \mathrm{~h}$ at 520,560, 610 and $650{ }^{\circ} \mathrm{C}$, respectively. For the preparation of the 200-nm PG sample, glass II was thermally treated for $24 \mathrm{~h}$ at $670{ }^{\circ} \mathrm{C}$. All heat treatments were conducted in a Nabertherm N7/H electrical furnace. The tempered glass block was then cut into slices of square shape $(10 \times 10 \times 0.5 \mathrm{~mm})$. To generate porosity, all membranes were leached with $\mathrm{HCl}\left(3 \mathrm{~mol}^{-1}\right)$ for $3 \mathrm{~h}$ at $90{ }^{\circ} \mathrm{C}$ under constant stirring (100 rpm) washed with deionized water until neutral, dried and, subsequently, leached in $\mathrm{NaOH}$ solution $(0.5 \mathrm{M})$ for $2 \mathrm{~h}$ at room temperature. Finally, the samples were washed again with deionized $\mathrm{H}_{2} \mathrm{O}$ until neutral and dried. The porous glass samples were denoted as $\operatorname{PG}(X)$, where $X$ represents the median pore width.

\section{Nitrogen sorption}

The pore width distribution of sample PG(15) and all parameters of the specific surface area were characterized by nitrogen sorption measurements. The measurements were taken at $-196{ }^{\circ} \mathrm{C}$ using a Quantachrome Autosorb-iQ device. The samples were degassed under vacuum at $200{ }^{\circ} \mathrm{C}$ for $12 \mathrm{~h}$ prior to the measurement. The specific surface area was determined using the model of Brunauer, Emmett and Teller (BET) in a relative pressure range of $p /$ $p_{0}=0.05-0.30$. The experimental error in the specific surface area was estimated to be approximately $5 \%$. The pore width distribution was calculated using the density functional theory (DFT) equilibrium kernel for $\mathrm{N}_{2}$ on $\mathrm{SiO}_{2}$ surfaces.

\section{Mercury intrusion porosimetry}

Pore widths, pore width distributions and pore volumes have been obtained by mercury intrusion porosimetry performed using a POROTEC Pascal $140 / 440$ device. Prior to the measurements, the samples were dried at $120{ }^{\circ} \mathrm{C}$ for $8 \mathrm{~h}$ and degassed for $20 \mathrm{~min}$. The experiments were performed in the pressure range from vacuum to 4000 bar. The pore widths were calculated using the Washburn equation [20] by assuming a cylindrical pore model, a contact angle of $141.3^{\circ}$ and a surface tension of $0.484 \mathrm{~N} / \mathrm{m}$ (cf. DIN 66133). The experimental error in the obtained pore volume was in the range between 3 and $10 \%$ for the calculated median pore width (Table 1).

\section{ASA-PG composites}

Acetylsalicylic acid (ASA) is a solid crystalline material with the habit of fine needles. It melts with decomposition at temperatures between 138 and $148{ }^{\circ} \mathrm{C}$. It is sparingly soluble in water, but dissolves very well in ethanol and chloroform at $20^{\circ} \mathrm{C}$ [21]. The synthesis of the composites was carried out at room temperature by dissolution of ASA in ethanol. At this temperature, the viscosity of ethanol is the lowest one, which ensures the smaller surface tension [22]. ASA crystals and ethanol were mixed until a saturated solution was obtained. Porous matrices with different pore widths (15-200 nm) were immersed in this solution and left for $1 \mathrm{~h}$. Subsequently, the matrices were removed, dried at $50{ }^{\circ} \mathrm{C}$ for $30 \mathrm{~min}$ and polished to remove the ASA crystallites from the surface. This process was repeated three times to ensure better pores filling [23, 24].

\section{Raman spectroscopy}

Raman spectra were recorded in backscattering geometry using Bruker FT-Raman RFS 100/S spectrometer and 1064-nm excitation. The spectral resolution was set to $2 \mathrm{~cm}^{-1}$. 
Table 1 Textural properties of the PG glass matrices

\begin{tabular}{lcclc}
\hline Sample & Median pore width $(\mathrm{nm})$ & BET surface $\left(\mathrm{m}^{2} \mathrm{~g}^{-1}\right)$ & Pore volume $\left(\mathrm{cm}^{3} \mathrm{~g}^{-1}\right)$ & Porosity (\%) \\
\hline PG(15) & 15 & 135 & $0.48 \pm 0.02$ & 51 \\
PG(25) & 25 & 49 & $0.38 \pm 0.02$ & 45 \\
PG(65) & 65 & 25 & $0.43 \pm 0.02$ & 49 \\
PG(90) & 90 & 13 & $0.38 \pm 0.04$ & 45 \\
PG(200) & 200 & 10 & $0.47 \pm 0.01$ & 51 \\
\hline
\end{tabular}

\section{Positron annihilation lifetime (PAL)}

PAL spectra were recorded using the fast coincidence system (ORTEC) of 230 ps resolution (full width at half maximum of a single Gaussian, determined by measuring the ${ }^{60} \mathrm{Co}$ isotope) at a temperature of $T=18{ }^{\circ} \mathrm{C}$ and relative humidity of $\mathrm{RH}=35 \%$. Each PAL spectrum was measured with a channel width of 24.8 ps (total number of channels 8196) and contained $6 \times 10^{6}$ coincidences in total. Isotope ${ }^{22} \mathrm{Na}$ (activity $100 \mathrm{kBq}$ ) used as a source of positrons (prepared from aqueous solution of ${ }^{22} \mathrm{NaCl}$, wrapped with Kapton ${ }^{\circledR}$ foil of $12 \mu \mathrm{m}$ thickness and sealed) was sandwiched between two identical samples. Before the measurements, the investigated samples were dried at $120{ }^{\circ} \mathrm{C}$ for $4 \mathrm{~h}$ in vacuum. All the PAL spectra were decomposed into five discrete exponentials $s(t)=\sum\left(I_{i} / \tau_{i}\right) \exp \left(-t / \tau_{i}\right) \quad$ with average positron lifetime $\tau_{i}$ and intensity $I_{i}$ using standard LT 9.0 program [25]. The results of decomposition are given in Table $\mathrm{S} 1$.

\section{Differential scanning calorimetry}

The heat-flow thermograms were measured using a high-resolution $(0.04 \mu \mathrm{W})$ Mettler Toledo DSC-1 calorimeter. The measurements were performed in the temperature range from -73 to $177^{\circ} \mathrm{C}$ (c.f. Figure S1) and a heating/cooling rate of $10{ }^{\circ} \mathrm{C} / \mathrm{min}$. Before the measurements, the calorimeter was calibrated to guarantee high-temperature accuracy of $\pm 0.2{ }^{\circ} \mathrm{C}$.

\section{Results and discussion}

\section{Characterization of the pore system of the PG matrix}

The pore width distributions of the PG matrices are depicted in Figs. 1 and 2. The textural properties are summarized in Table 1.
It should be mentioned that all porous glass matrices used in the experiment were characterized by a uniform pore width distribution. The pore width could have been varied in the broad range between 15 and $200 \mathrm{~nm}$. It is clear from Table 1 that as expected, the specific surface area decreases with increasing median pore width. Due to the porous glasses formation process, the pore volume and thus the porosity remain constant at $0.5 \mathrm{~cm}^{3} / \mathrm{g}$ and $50 \%$, respectively. This is the very important observation for the systematic investigation of confinement effects. In the case of complete pore filling, the amount of the embedded material remains constant. Only the dimension of the pores is varied.

\section{Positron annihilation lifetime (PAL)}

Usually, in porous materials similar to those studied in the present work, from the decomposition of the PAL spectra, up to four/five components representing different annihilation sites can be well resolved. The presence of the fifth component in data obtained for the analyzed samples is evident as it was impossible to obtain a regular distribution of standard deviations in the case of deconvolution of spectra into four components.

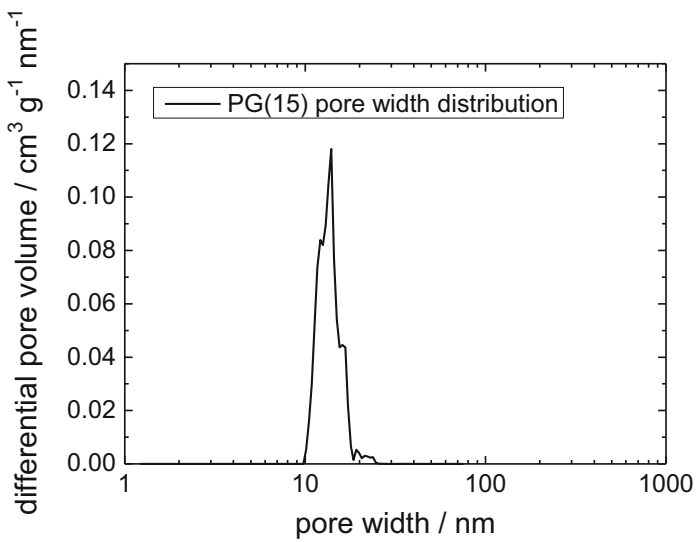

Figure 1 Pore width distribution of PG(15) calculated by the DFT equilibrium method from nitrogen sorption data. 


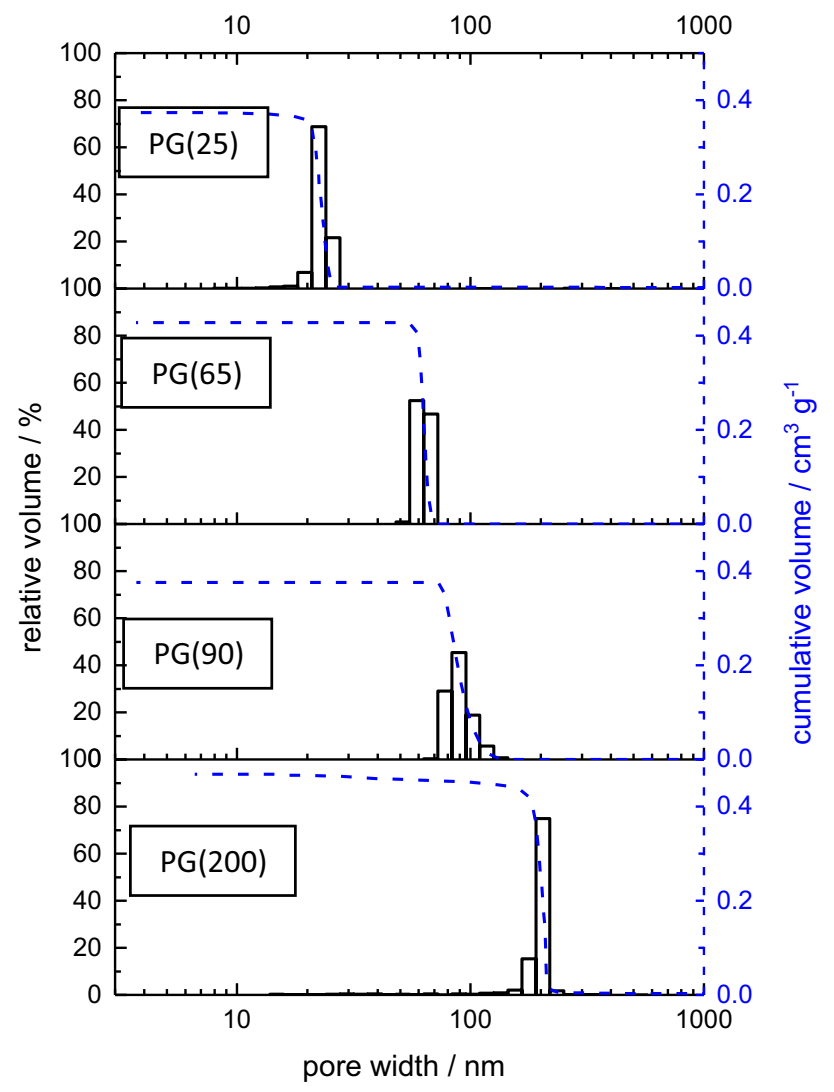

Figure 2 Pore width distributions (bars) and mercury intrusion curves (blue dashed curves) of PG(25), PG(65), PG(90) and PG(200) calculated by the cylindrical pore model from mercury intrusion data. Bars indicate the relative pore volume, and dashed line indicates the cumulative pore volume.

An estimation of the dimension of the open volumes of the investigated samples can be obtained by the Tao-Eldrup semiempirical model $[26,27]$ that relates the ortho-positronium $(o-P s)$ lifetime reduced by pick-off process to the average sizes of the void volume. The cavity hosting $o-P S$ is assumed to be a spherical void with effective radius $R$. Such an $o-P S$ trap has a potential well with finite depth. However, for the convenience of calculations one usually assumes the potential well depth as infinite, but the radius increased to $R+\Delta R(0.166 \mathrm{~nm})$. The radius is assumed to be an empirical parameter which describes the penetration of the $o-P S$ wave function into the bulk [34]. The relationship between the $o-P S$ lifetime $\tau_{i}$ and the radius $R_{i}$ is the following:

$\tau_{i}=\frac{1}{2}\left[1-\frac{R_{i}}{R_{i}+\Delta R}+\frac{1}{2 \pi} \sin \left(\frac{2 \pi R_{i}}{R_{i}+\Delta R}\right)\right]^{-1}$
The values $\tau_{i}$ and $R_{i}$ (hole radius) are expressed in ns and $\AA$, respectively. $\Delta R=1.66 \AA$ is determined by fitting of the experimental values of $\tau$ obtained for the materials with known hole size.

The values of $\tau_{i}$ and the corresponding free volume radii $\left(R_{i}\right)$ for the three longest components can be used for determination of the percentage share of the free volume fraction $\left(f_{\mathrm{V}}\right)$ according to the equation:

$f_{\mathrm{v} i}=A \cdot I_{i}(\%) \cdot\left(\frac{4}{3} \pi R_{i}^{3}\right)$

Although the value of $A$ constant in the above formula is known exactly for polymers (0.0018) $[28,29]$, it was used in the study to obtain free volume fraction in the tested porous materials, assuming that the results are subjected to uncertainties associated with the adopted procedure. Also, the values of the radii obtained from Eq. (1) should be interpreted only as rough estimates, since real voids are irregularly shaped. The results of calculation of radii of spherical voids and their fraction for the $o-P s$ components are given in Table S3.

In the PG (65) matrix material, three types of spherical voids with diameters of $0.512 \mathrm{~nm}, 1.22 \mathrm{~nm}$ and $4.22 \mathrm{~nm}$ were identified. After impregnation with ASA, the small pores remained unchanged, while the diameters of the largest pores decreased from $4.22 \mathrm{~nm}$ to $3.56 \mathrm{~nm}$. Furthermore, their fraction decreased from 57.8 to $28.7 \%$. Changes in distributions of free volume fraction $\left(\mathrm{d} f_{\mathrm{v}} / \mathrm{d} D\right)$ for all three $o-P S$ components, before and after doping by ASA, are illustrated in Fig. 3.

The reduction in the diameter of the largest of the spherical holes available for o-Ps-atoms in the ASAPG(65) composite indicates their partial filling by the admixture of ASA. In addition, for more than $50 \%$ of these holes the filling has taken place to such an extent that it was impossible to create an $o-P s$ in them. The large value of the fraction $\left(f_{\mathrm{v}}\right)$ for these holes, compared to the fractions for the other components, is not related to the number of holes but to their size. The lack of significant changes within the first two $o$ $P s$ components after impregnation suggests that the ASA could not intrude these pores.

\section{Raman spectroscopy}

Figure 4 presents Raman spectra for pure crystalline ASA I polymorph [14] and the ASA-PG composites with pores ranging from 15 to $200 \mathrm{~nm}$. The spectrum 


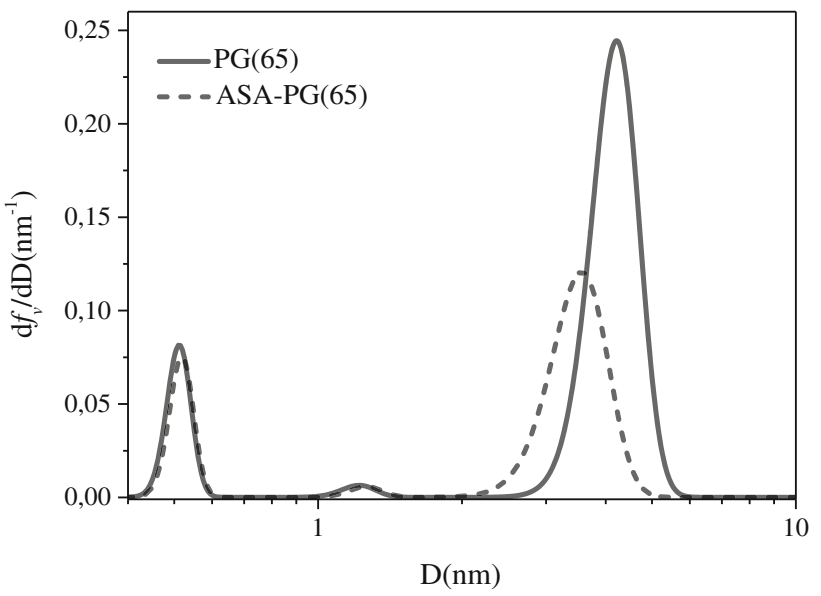

Figure 3 Distributions of free volume fraction $\left(\mathrm{d} f_{\mathrm{v}} / \mathrm{d} D\right)$ for all three $o$-PS components, before and after doping by ASA. The area under curves is normalized to the free volume fractions $\left(f_{\mathrm{v}}\right)$ for particular components.

of ASA-PG(15) shows the presence of narrow bands at 1086, 712, 282 and $155 \mathrm{~cm}^{-1}$ and broad bands at 2904, 1438, 610 and $447 \mathrm{~cm}^{-1}$. They corresponds to glass matrix containing borium/silicone polyhedra or rings [30] and/or the residues of ethyl alcohol (solvent) used for the ASA dissolution. The ASA spectrum shows that the most intense peaks are located at 3092, 3077, 2941, 1606, 1292, 1192, 1044, 752, 172, 132 and $120 \mathrm{~cm}^{-1}$. They were used as probes to study the presence and properties of ASA embedded in the PG matrices. The normalized spectra collected for samples with different pore widths show that the signal coming from ASA is increasing with increasing pore

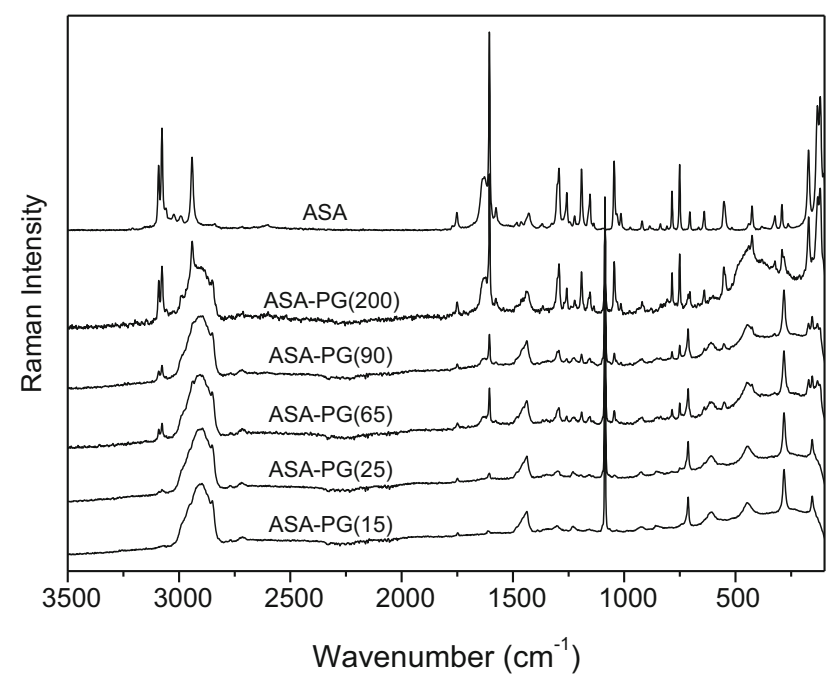

Figure 4 Details of Raman spectra of ASA embedded into porous glass matrices with different pore sizes. widths. It can be clearly seen that the signal of ASA can be still detected even for the lowest pore widths, i.e., $15 \mathrm{~nm}$. The detailed analysis of ASA bands in glasses allows one to draw further conclusions. Firstly, the FWHMs (full width at half maximum) of observed ASA bands confined in PG remains relatively low even for ASA-PG(65) sample. For ASAPG(15), which possesses the smallest pores, the ASA signal is observed to be very weak and the corresponding FWHMs increases likely due to the size effects, i.e., phonon confinement effect, creation of defects, distribution of crystallite size and/or change in lattice parameters (strain) [31]. It is well known that bands become asymmetric and broad for very small nanocrystallites. It supports our conclusion that confined ASA probably remains in crystalline form in all studied samples. Secondly, the number of the strongest bands corresponding to lattice modes of ASA is unchanged for pores with average size ranging from 65 to $200 \mathrm{~nm}$. It suggests that confined ASA does not change its symmetry at least down to $65 \mathrm{~nm}$. Thirdly, the strongest band observed for pure ASA at $1606 \mathrm{~cm}^{-1}$ remains in the same position when pores are decreased to $65 \mathrm{~nm}$. Their further lowering to 25 and $15 \mathrm{~nm}$ causes up-shift to 1607 and $1610 \mathrm{~cm}^{-1}$, respectively. The bands observed at 1752 and $1045 \mathrm{~cm}^{-1}$ shift slightly toward lower wavenumbers, i.e., 1748 and $1038 \mathrm{~cm}^{-1}$. This behavior proves that the interactions between matrix and confined ASA crystallites are stronger in the smallest cavities and can be explained by the size effects in the smallest crystallites of confined ASA.

\section{Differential scanning calorimetry}

Melting temperature of ASA is observed for all samples, but the endothermic peak splits into two and shifts to significantly lower temperatures as the pore width $d$ decreases. For ASA confined into nanopores with the largest $d$ value of $200 \mathrm{~nm}$, the first peak $T_{1}$ at $131{ }^{\circ} \mathrm{C}$ is significantly below the bulk melting phase of polymorph I at $144.9{ }^{\circ} \mathrm{C}$ but only slightly below that of polymorph II at $135.5^{\circ} \mathrm{C}$ (c.f. Figure 5). The decrease in the pore width $d$ to $25 \mathrm{~nm}$ shifts $T_{1}$ to about $112{ }^{\circ} \mathrm{C}$. The ASA spatial confinement leads to the appearance of additional peak. Similarly to the $T_{1}$, also the melting temperatures $T_{2}$ shifts to the lower temperature, from 124 to $95{ }^{\circ} \mathrm{C}$, with decreasing pore sizes. This unambiguously confirms that the spatially confined ASA prefers less 
energetically stable states (dashed areas in Fig. 5). It is worth noting that the reduction in ASA crystallite sizes causes both the smearing of melting temperatures and the decrease in specific heat excess. This undoubtedly confirms that the spatially confined ASA prefers less energetically stable states. This fact linked together with the observed significant changes in melting temperature for the higher $d$ leads to the conclusion that ASA confined in a matrix crystallizes in the II polymorph structure. This fact indicates that the polymorph II is less energetically stable [32]. It should be mentioned that the assumption that ASA is crystalline in all the studied samples is not fully supported by performed analysis. Structural investigations, for instance XRD measurements, could be conducted to clarify this issue.

It is well known that by assuming equivalence of the free energies of the confined liquid and crystalline phases at $T_{\mathrm{M}}$ the confinement-induced melting temperature shift may be determined $[33,34]$. The $T_{M}(d)$ temperature of crystals confined to cylindrical pores can be calculated using the following Gibbs-Thomson equation [35]:

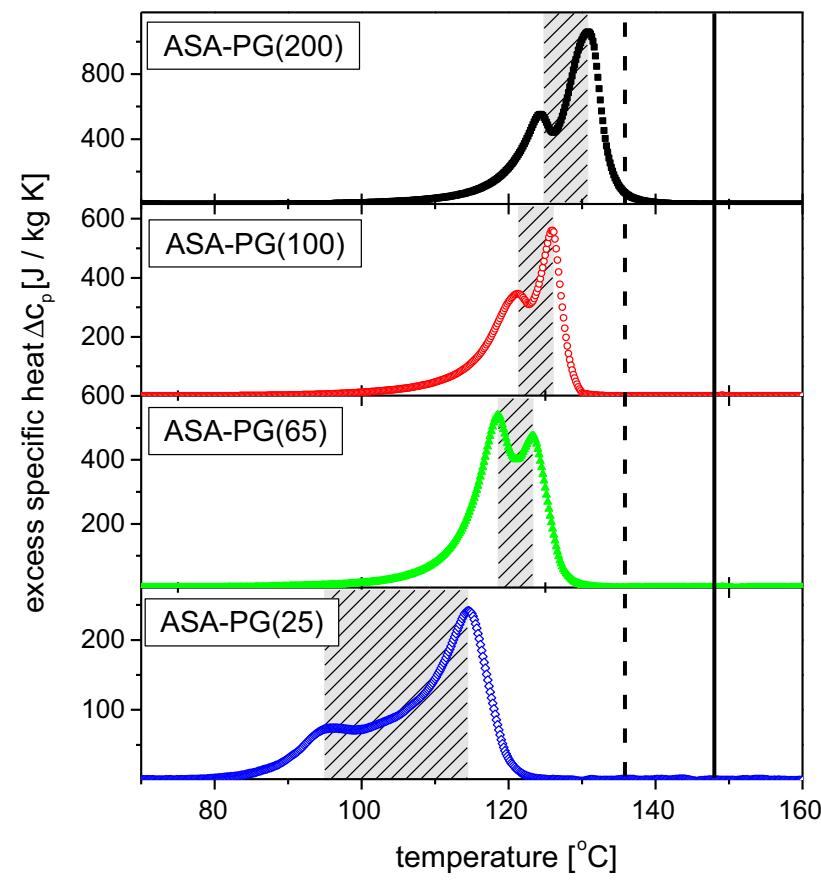

Figure 5 DSC heating scans obtained at the rate $\mathrm{d} T / \mathrm{d} t=10 \mathrm{~K} /$ min for ASA-PG composites with different pore width. Solid line at $148{ }^{\circ} \mathrm{C}$ denotes the melting temperature for ASA I polymorphic phase, while dashed line represents the melting temperature for ASA II.
$T_{\mathrm{M}}(d)=T_{\mathrm{M}}(\infty) \cdot\left[1-4 \cdot \frac{\sigma_{\mathrm{cl}}}{d \cdot \Delta H_{\mathrm{M}} \cdot \rho_{\mathrm{c}}}\right]$

where $\sigma$ denotes the surface tension between ASA nanocrystals and liquid phases, $\Delta H_{\mathrm{M}}$-the melting heat and $\rho$-the density. For both the sets of melting temperatures, $T_{\mathrm{M}}$ shifts linearly with $1 / d$, as predicted by Gibbs-Thomson equation, and linear extrapolations to bulk $d$ revealed $T_{M}(\infty)$. The linear extrapolation of the data points obtained from the observed DSC spectra yields, however, a $T_{(\infty)}$ value of about $133.0 \pm 1.8{ }^{\circ} \mathrm{C}$, which is significantly lower than the bulk melting temperature of ASA form I $\left(T_{M, I}(\infty)=144.9^{\circ} \mathrm{C}\right)$. We suppose that isothermal crystallization of ASA confined to PGs at room temperatures provides nearly exclusively form II, similarly to the results for acetaminophen which isothermally crystallized in less stable form III [36]. Moreover, the Gibbs-Thomson plot (Fig. 6) shows coexistence of two polymorphic phase of ASA confined into PG.

To check whether the induced intermediate phase is pressure-induced ASA III polymorph resulting from the different thermal expansion coefficients of embedded materials and PG matrix, the pressure effect in these composites was estimated using the following formula [37]:

$p^{\prime}=\frac{B \cdot B_{\mathrm{M}} \cdot \Delta T\left(2 \alpha_{1}+\alpha_{3}-3 \alpha_{\mathrm{M}}\right)}{B+B_{\mathrm{M}}}$

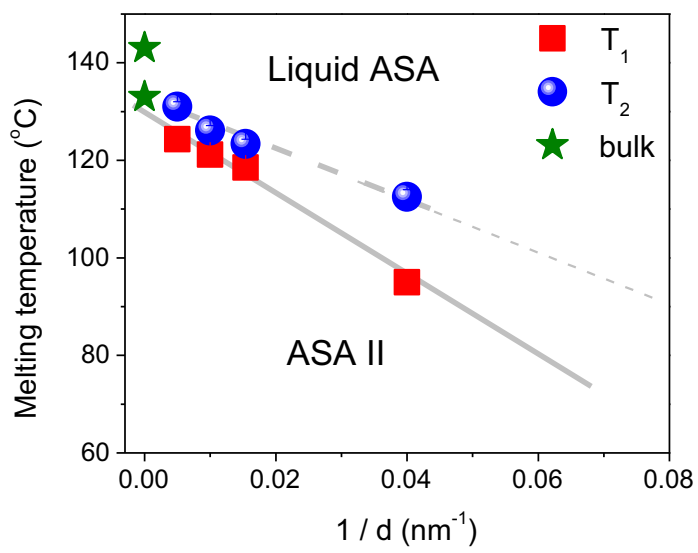

Figure 6 The dependence of melting temperatures versus the inverse of the pores width $\mathrm{d}^{-1}$. The $T_{1}$ and $T_{2}$ values are related to the maximum of the melting peaks in the corresponding DSC heating scans. Bulk melting temperatures (stars) of bulk ASA I and II are given for comparison. The lines represent linear fits to the experimental data $\left(T_{\mathrm{M}, T 1 \text {-bulk }} \approx 132{ }^{\circ} \mathrm{C} ; \quad T_{\mathrm{M}, T 2 \text {-bulk }}\right.$ $\approx 133{ }^{\circ} \mathrm{C}$ ). 
where $\Delta T$ denotes the temperature difference, $\alpha_{M}$ and $\alpha$ are thermal expansion coefficients of the porous glass matrix and the ASA, and $\mathrm{B}_{\mathrm{M}}$ and $\mathrm{B}$ denote the bulk modulus for the porous matrix and the ASA, respectively.

To simplify the calculations, a complete (100\%) filling of the pores was assumed. Application of the proposed approach allowed to estimate the order of magnitude of the pressure present within the obtained composite. In calculations, the following input data (appropriate for ASA) were used $\left(B=13.80 \quad \mathrm{GPa} \quad[38] ; \quad \alpha_{1}=92.6(7) \times 10^{-6} \mathrm{~K}^{-1}\right.$, $\alpha_{3}=80.4(2) \times 10^{-6} \mathrm{~K}^{-1}$ [39]) and for glass $\mathrm{SiO}_{2} \mathrm{~B}_{2} \mathrm{O}_{3}$ $\mathrm{Na}_{2} \mathrm{O}\left(B_{\mathrm{M}}=206 \times 10^{9} \mathrm{~Pa} ; \alpha_{\mathrm{M}}=3.5 \times 10^{-6} \mathrm{~K}^{-1}[40]\right.$. Assuming that $\Delta T=115 \mathrm{~K}$, the inner pressure in the composite was estimated to be equal to $0.4 \pm 0.1 \mathrm{GPa}$. The assessed value of pressure created by the PG matrix on embedded ASA crystallites is smaller than the pressure which would induce the polymorphic phase III, i.e., about 2GPa [16]. However, taking into account that these results were obtained for the bulk ASA I not for the ASA II, it is reasonable to assume that the new induced phase is an ASA III. This assumption, however, requires additional experimental investigations.

\section{Conclusions}

In summary, we have analyzed the DSC data combined with the Raman and PALS spectra of acetylsalicylic acid (ASA)-porous glass (PG) composites. The obtained results provide an evidence that ASA confined in a matrix prefers to crystallize in II polymorph-type structure. Moreover, it was shown that the reduction in ASA crystallite sizes causes smearing of both melting temperatures and the decrease in specific heat excess. It should be pointed out that the predictions of pressure effect on embedded crystallites may indicate that the spatial confinement of ASA leads to the appearance of ASA III polymorph.

Yet, the reasons for the stabilization of metastable ASA polymorph II in nanoconfinement are not fully understood and require further studies.

Crystallization of ASA confined to nanoporous systems will enable detailed characterization of its metastable polymorphic forms, resulting potentially in better, controllable release characteristics of a next generation of drug delivery systems.

\section{Acknowledgements}

A.S. gratefully acknowledges financial support from Wrocław University of Science and Technology.

\section{Compliance with ethical standards}

Conflict of interest The authors declare that they have no conflict of interest.

Electronic supplementary material: The online version of this article (https://doi.org/10.1007/s108 53-018-2853-8) contains supplementary material, which is available to authorized users.

Open Access This article is distributed under the terms of the Creative Commons Attribution 4.0 International License (http://creativecommons.org/ licenses/by/4.0/), which permits unrestricted use, distribution, and reproduction in any medium, provided you give appropriate credit to the original author(s) and the source, provide a link to the Creative Commons license, and indicate if changes were made.

\section{References}

[1] Singco B, Liu L, Chen Y, Shih Y, Hauang H, Lin C (2016) Approaches to drug delivery: confinement of aspirin in MIL$100(\mathrm{Fe})$ and aspirin in the de novo synthesis of metal-organic frameworks. Microporous Mesoporous Mater 223:254-260

[2] Tawashi R (1968) Aspirin: dissolution rates of two polymorphic forms. Science 160:76-78

[3] Olmsted JA III (1998) Synthesis of Aspirin: a general chemistry experimental. J Chem Educ 75:1262-1263

[4] Eldrup M, Lightbody D (1981) The temperature dependence of positron lifetimes in solid pivalic acid. J Chem Phys 63:51-58

[5] Beiner M (2008) Nanoconfinement as a tool to study early stages of polymer crystallization. Part B Polym Phys 46:1556-1561

[6] Ouvrard C, Price SL (2004) Toward crystal structure prediction for conformationally flexible molecules: the heasaches illustrated by aspirin. Cryst Growth Des 4:1119-1127

[7] Vishweshwar P, McMahon JA, Oliviera M, Peterson ML, Zaworotko MJ (2005) The predictably elusive form II of aspirin. J Am Chem Soc 127:16802-16803

[8] Brela MZ, Wójcik MJ, Witek ŁJ, Bocza M, Wroca E, Hashim R, Ozaki Y (2016) Born-Oppenheimer molecular dynamics study on proton dynamics of strong hydrogen 
bonds in aspirin crystals, with emphasis on differences between two crystal forms. J Phys Chem B 120:3854-3862

[9] Morsy R, Elsayed M, Rehberg RK, Dlubek G, Elnimr T (2010) Positron annihilation spectroscopic study of hydrothermally synthesized fine nanoporous hydroxyapatite agglomerates. J Eur Ceram Soc 30:1897-1901

[10] LeBlanc LM, Otero-de-la-Roza A, Johnson ER (2016) Evaluation of shear-slip transitions in crystaline aspirin by density- functional theory. J Cryst Growth 16:6867-6873

[11] Bond AD, Solanko KA, Parsons S, Redder S, Boese R (2011) Single crystals of aspirin form II: crystallization and stability. CrystEngComm 13:399-401

[12] Bond AD, Boese R, Desiraju GR (2007) On the polymorphism of aspirin: crystalline aspirin as intergrowths of two “polymorphic” domains. IntesScience 46:618-622

[13] Beckert S, Gratz M, Kullmann J, Enke D, Stallmach F (2013) Concentration-dependent self-diffusion of water in aqueous solutions of lithium chloride confined to porous glasses. Appl Magn Reason 44:827-836

[14] Shtukenberg AG, Hu ChT, Schmidt MU, Xu W, Tan M, Kahr B (2017) The third ambient aspirin polymorph. J Cryst Growth 17:3562-3566

[15] Zhu Q, Shtukenberg AG, Carter DJ, Yu TQ, Yang J, Chen M, Raiteri P, Oganov AR, Pokroy B, Polishchuk I, Bygrave PJ, Day GM, Rohl AL, Tuckerman ME, Kahr BJJ (2016) Resorcinol crystallization from the melt: a new ambient phase and new "riddles". Am Chem Soc 138:4881-4889

[16] Crowell EL, Dreger ZA, Gupta YM (2015) High-pressure polymorphism of acetylsalicylic (aspirin): Raman spectroscopy. J Mol Struct 1082:29-37

[17] Varughese S, Kiran MSRN, Solanko KA, Bond AD, Rammurty U, Desiraju GR (2011) Interaction anisotropy and shear instability of aspirin polymorphs established by nanoidentation. Chem Sci 2:2236-2242

[18] Hamilton BD, Ha JM, Hillmyer MA, Ward MD (2012) Manipulating crystal growth and polymorphism by confinement in nanoscale crystallization chambers. Acc Chem Res 45:414-423

[19] Rengarajan GT, Enke D, Steinhart M, Beiner M (2008) Stabilization of the amorphous state of pharmaceuticals in nanopores. J Mater Chem 18:1537-2539

[20] Washburn EW (1921) Note on a method of determining the distribution of pore sizes in a porous material. Proc Natl Acad Sci USA 7(4):115-116

[21] Schror K (2009) Acetylsalicylic acid, 1st edn. Wiley, New York, pp 375-377

[22] Bica K, Rijksen C, Nieuwenhuyzen M, Rogers RD (2010) In search of pure liquid salt forms of aspirin: ionic liquid approaches with acetylsalicylic acid and salicylic acid. Phys Chem Chem Phys 12:2011-2017
[23] Poprawski R, Rysiakiewicz-Pasek E, Sieradzki A, Ciżman A, Polańska J (2007) Ferroelectric phase transitions in KNO3 embedded into porous glasse. J Non-Cryst Sol 353:4457-4461

[24] Sieradzki A, Komar J, Rysiakiewicz-Pasek E, Ciżman A, Poprawski R (2010) Calorimetric investigations of phase transitions in $\mathrm{KNO} 3$ embedded into porous glasses. Ferroelectrics 402:60-65

[25] Kansy J (1996) Microcomputer program for analysis of positron annihilation lifetime spectra. Nucl Instr Methods A 374:235-244

[26] Tao SJ (1972) Positronium annihilation in molecular substances. J Chem Phys 56:5499-5510

[27] Eldrup M, Lightbody D, Sherwood JN (1981) The temperature dependence of positron lifetimes in solid pivalic acid. Chem Phys 63:51-58

[28] Zhang Y, Li R, Jean YC (2003) Free-volume data in polymeric materials. World Scientific, Singapore, pp 373-374

[29] Wang YY, Nakanishi H, Jean YC (1990) Positron annihilation in amine-cured epoxy polymers-pressure dependence. J Polym Sci Part B Polym Phys Ed 28:1431-1441

[30] Furukawa T, White WB (1981) Raman spectroscopic investigation of sodium borosilicate glass structure. J Mater Sci 16:2689-2700

[31] Mączka M, Ptak M, Kurnatowska M, Kępiński L, Tomaszewski P, Hanuza J (2011) Phonon properties of nanosized MnWO4 with different size and morphology. J Sol State Chem 184:2446-2457

[32] Reilly AM, Tkatchenko A (2014) Role of dispersion interactions in the polymorphism and entropic stabilization of the aspirin crystal. Phys Rev Lett 113:055701-1-055701-5

[33] Dwyer LM, Michaelis VK, O'Mahony M, Griffin RG, Myerson AS (2015) Confined crystallization of fenofibrate in nanoporous silica. CrystEngComm 17:7922-7929

[34] Zinn T, Willner L, Lund R (2014) Nanoscopic confinement through self-assembly: crystallization within micellar cores exhibits simple Gibbs-Thomson behavior. Phys Rev Lett 113:238305-1-238305-5

[35] Alcoutlabi M, McKenna GB (2005) Effects of confinement on material behaviour at the nanometre size scale. J Phys Condens Matter 17:R461-R524

[36] Beiner M, Rengarajan GT, Pankaj S, Enke D, Steinhart M (2007) Manipulating the crystalline state of pharmaceuticals by nanoconfinement. Nano Lett 7:1381-1385

[37] Marciszyn T, Poprawski R, Komar J, Sieradzki A (2010) Phase transition in $\mathrm{NH}_{4} \mathrm{H}_{2} \mathrm{PO}_{4}$ : porous glass composites. Phase Transit 83:909-916

[38] Payne RS, Roberts RJ, Rowe RC, McPartlin M, Bashal A (1996) The mechanical properties of two forms of primidone 
predicted from their crystal structures. Int $\mathrm{J}$ Pharm 145:165-173

[39] Bauer JD, Haussuhl E, Winkler B, Arbeck D (2010) Elastic properties, thermal expansion, and polymorphism of acetylsalicylic acid. Cryst Growth Des 10:3132-3140
[40] Carlotti G, Doucet L, Dupeux M (1996) Comparative study of the elastic properties of silicate glass films grown by plasma enhanced chemical vapor deposition. J Vac Sci Technol B 14(6):3460-3464 